EPJ Web of Conferences 109, 01001 (2016)

DOI: $10.1051 /$ epjconf/201610901001

(C) Owned by the authors, published by EDP Sciences, 2016

\title{
Explosive Nuclear Burning in the pp-Chain Region and the Breakout Processes
}

\author{
S. Kubono ${ }^{1,2,3 a}$, H. Yamaguchi ${ }^{3}$, S. Hayakawa ${ }^{3}$, S.Q. $\mathrm{Hou}^{2}$, J.J. He ${ }^{2}$ \\ ${ }^{1}$ RIKEN Nishina Center, 2-1 Hirosawa, Wako, 351-0198 Saitama, Japan \\ ${ }^{2}$ Institute of Modern Physics, CAS, Lanzhou, China \\ ${ }^{3}$ Center for Nuclear Study, University of Tokyo, 2-1 Hirosawa, Wako, 351-0198 Saitama, Japan
}

\begin{abstract}
The nuclear reactions in the pp-chain region and on the breakout process from the pp-chain region under very high temperature conditions are reviewed, and some possibilities for experimental investigation are discussed. The reactions discussed could play an important role typically for the primordial nucleosynthesis and supernova nucleosynthesis. Specifically, I discuss here the reactions starting from the two key nuclei, ${ }^{7} \mathrm{Be}$ and ${ }^{7} \mathrm{Li}$. The ${ }^{7} \mathrm{Be}(\mathrm{n}, \alpha)$ reaction, which destroys ${ }^{7} \mathrm{Be}$, is considered to have a large impact to the primordial ${ }^{7} \mathrm{Li}$ problem. Our recent estimate of the reaction rate indicates that the reaction rate can be about one order of magnitude smaller than the rate currently adopted, suggesting this channel has a minor effect for the ${ }^{7} \mathrm{Li}$ problem. Under a proton-rich environment at high temperature like the vp-process, the ${ }^{7} \mathrm{Be}(\alpha, \gamma){ }^{11} \mathrm{C}(\alpha, \mathrm{p}){ }^{14} \mathrm{~N}$ pathway is expected to play a majpr role for heavy element synthesis, comparable to the triple alpha process. These two reactions on the pathway were investigated by using low-energy, high-intensity RI beams of ${ }^{7} \mathrm{Be}$ and ${ }^{11} \mathrm{C}$. The results support the theoretical prediction of heavy nucleus production at around mass $90-100$ by the vp-process, where the anomalously abundant p-nuclei exist. The reactions on the breakout sequence of ${ }^{7} \operatorname{Li}(\mathrm{n}, \gamma){ }^{8} \operatorname{Li}(\alpha, \mathrm{n}){ }^{11} \mathrm{~B}$ are also discussed which could paly a crucial role in nuetron-rich envirnments, like in the primirdial universe as well as the early stage of the r-process. The cross sections of the first step reaction ${ }^{7} \mathrm{Li}(\mathrm{n}, \gamma)^{8} \mathrm{Li}$ seems well confirmed, but the second step reaction ${ }^{8} \operatorname{Li}(\alpha, n){ }^{11} \mathrm{~B}$ still is not well known yet, whose status of the study is discussed.
\end{abstract}

\section{The pp-Chain Region and the Breakout Process}

Observation of elements is one of the keys for studying the evolution of the universe as well as various stellar phenomena. Almost all the elements were produced by nuclear reactions along the evolution of the universe. Thus, studying nucleosynthesis is a powerful tool to look back the history of the universe [1], and investigate the mechanism of the evolution.

The primordial nucleosynthesis just after the big bang is one of the key elements that support the big bang model. The nuclear reactions there took place in a neutron-rich environment under relatively high temperature conditions for the first few minutes just after the big bang, and produced primarily the nuclides in the pp-chain region. Thus, the nuclear reactions in the pp-

\footnotetext{
${ }^{a}$ Corresponding author: kubono@riken.jp
} 
chain region played crucial role in the primordial nucleosynthesis. However, the recent observation of the cosmic microwave background by WMAP[2] and the ESA Plank Mission [3] together with the optical observations [4] have revealed that there is a significant inconsistency in the ${ }^{7} \mathrm{Li}$ abundance[5]. There still is a possibility that this problem comes from unstudied, or not well studied nuclear reactions in the pp-chain region in the primordial nucleosynthesis, although so much efforts have been made for many years in nuclear astrophysics.

The nuclear reactions in the pp-chain region also play a very important role in type II supernovae, because $\mathrm{Fe}$ and $\mathrm{Ni}$ nuclides in the core region may be dissociated to protons, neutrons and alphas, and the nucleosynthesis starts from the pp-chain region and produces heavier nuclei. Thus, the nucleosynthesis in the early epoch of type II supernovae first make the seed nuclei from the pp-chain region in the r-process and the vp process [6-8].

Thus, the nuclear reactions in the pp-chain region and on the breakout pathway from the ppchain region control significantly the production of heavy nuclides. There are a lot of efforts made for the reaction studies in the pp-chain region at low temperatures, for instance, for the solar neutrino problems, but only a few efforts for the reactions at high temperatures, where more unstable nuclei are involved.

It should be worth noting that major efforts were made for the proton capture reactions on proton-rich unstable nuclei in the proton-rich nuclear regions for explosive hydrogen burning. However, at high temperatures in the light mass region, alpha induced reactions play a dominant role, mostly $(\alpha, p)$ reactions that bypass the waiting points, and would accelerate the nucleosynthetic flow to heavier mass regions [9]. There are several efforts reported for these reactions, but almost none are known well yet. Another interesting point for the nucleosynthesis at high temperature in the proton-rich nuclear region is that neutron-induced reactions will also play an important role like in the vp-process. Of course, it is a very challenging subject to investigate such reactions because both the projectile and the target are unstable.

\section{$1.1{ }^{7} \mathrm{Li}$ problem in the primordial origin}

There are many astronomical observations which report that ${ }^{7} \mathrm{Li}$ together with other nuclides ${ }^{1} \mathrm{H},{ }^{2} \mathrm{H},{ }^{4} \mathrm{He}$, etc, may be of primordial origin, although there could be other contributions for ${ }^{7} \mathrm{Li}$ abundance. The elemental abundance calculations by the standard big bang model reproduce all the nuclides in the pp-chain region except for ${ }^{7} \mathrm{Li}$ [5]. There are two possibilities for this discrepancy; one possibility is due to some uncertainties in nuclear physics, and the other is the one in the astronomical observation. Thus, this problem should be worked out by checking all possible nuclear reactions relevant thoroughly for nuclear physics side. ${ }^{7} \mathrm{Li}$ is considered to have been produced primarily through the $\left.{ }^{3} \mathrm{H}(\alpha, \gamma)\right)^{7} \mathrm{Li}$ reaction and from ${ }^{7} \mathrm{Be}$. Figure 1 shows a typical calculation [10] of the primordial nucleosynthesis. The main contributor for ${ }^{7} \mathrm{Li}$ is considered to be the ${ }^{7} \mathrm{Be}(\mathrm{n}, \mathrm{p})^{7} \mathrm{Li}$ reaction, whereas the ${ }^{7} \mathrm{Be}(\mathrm{n}, \alpha)$ may be an important destruction reaction of ${ }^{7} \mathrm{Be}$, which would reduce the ${ }^{7} \mathrm{Li}$ production. Both reactions on ${ }^{7} \mathrm{Be}$, however, are not well investigated, especially almost none for the $(n, \alpha)$ reaction.

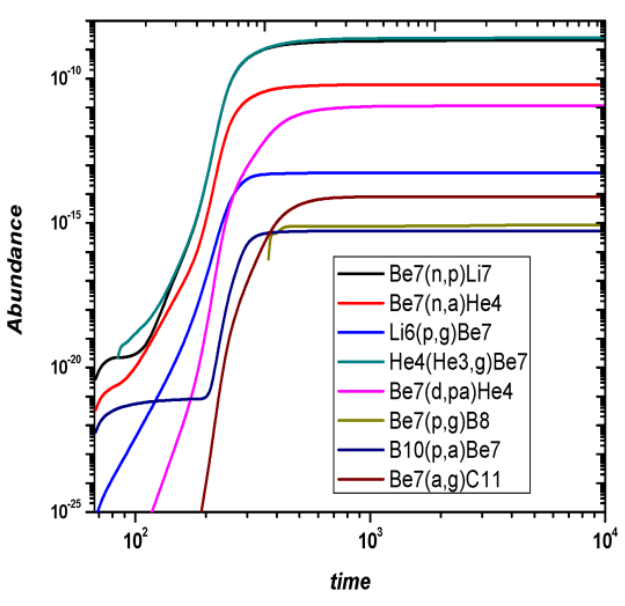

Figure 1 The nuclear reactions relevant to ${ }^{7} \mathrm{Be}$ in the primordial nucleosynthesis by the standard model [10]. 
In the pp-chain region, there remain some possibilities that some unknown resonances would affect the ${ }^{7} \mathrm{Li}$ abundance.

Another interesting reactions for the primordial nucleosynthesis are those on the possible pathway of the breakout from the pp-chain region, ${ }^{7} \operatorname{Li}(\mathrm{n}, \gamma){ }^{8} \mathrm{Li}(\alpha, \mathrm{n}){ }^{11} \mathrm{~B}[12]$. These reactions also play an important role in the early stage of the r-process, as will be discussed in sec. 3 [13].

\subsection{The nuclear reactions on the beakout processes under explosive burning at high temperatures}

The nuclear reactions in the pp-chain region is depicted in Fig. 2 together with possible breakout processes under high temperature astronomical conditions $[11,12,13]$. The triplealpha reaction is considered to be a key process for a wide temperature range in various scenarios. The ${ }^{4} \mathrm{He}(\alpha)^{8} \mathrm{Be}(\mathrm{n}, \gamma)^{9} \mathrm{~B}$ process is considered to be another important pathway in type II supernovae. In addition, the pathway, mentioned in the last subsec. ${ }^{7} \mathrm{Li}(\mathrm{n}, \gamma){ }^{8} \mathrm{Li}(\alpha, \mathrm{n}){ }^{11} \mathrm{~B}$ also is important in the type II supernovae, and the possible $\mathrm{CNO}$ element production in the primordial universe.

Under proton-rich, high temperature conditions like in the vp-process in the very early epoch of type II supernovae, nuclear reaction chains that involve proton-rich nuclei are considered to be favourable like ${ }^{7} \mathrm{Be}(\alpha, \gamma){ }^{11} \mathrm{C}(\alpha, p){ }^{14} \mathrm{~N}$, and ${ }^{7} \mathrm{Be}(\alpha, p){ }^{10} \mathrm{~B}(\alpha, p){ }^{13} \mathrm{C}$

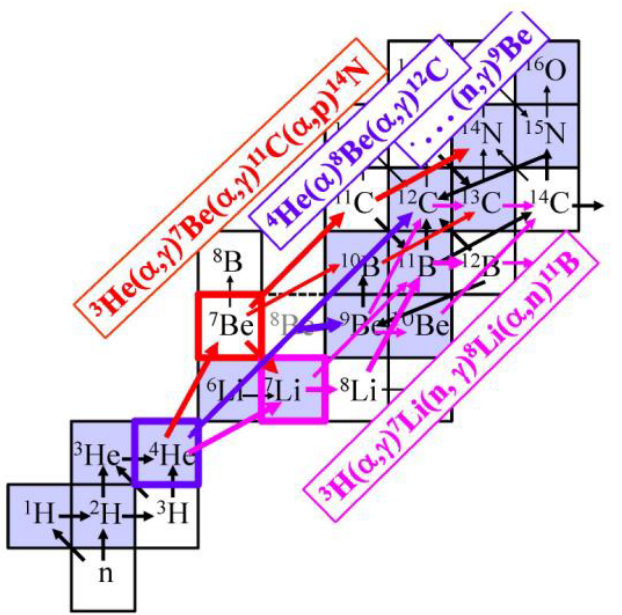

Figure 2 Schematic nucleosynthetic flows at very high temperatures in the pp-chain and breakout regions. [10]. Since the nuclear reactions on the breakout processes involve unstable nuclei, most of them were not investigated well yet. The breakout conditions actually influence very much the heavy element synthesis as we see in the next subsec. Therefore, the reaction study of these processes along the breakout processes is considered to be one of the key subjects for investigation of explosive burning in nuclear astrophysics. This paper will discuss some important reactions among them.

\subsection{The vp-process in type II supernovae}

The vp-process, proposed in 2006 [6-8], may take place at the very early epoch of type II supernovae in the ejecta near the inner core. Here, it can be proton-rich with some fraction of neutrons at very high temperatures because they are in equilibrium through the weak interaction; $v_{e}+n \leftrightarrow p+e^{-}, \quad \bar{v}_{e}+p \leftrightarrow n+e^{+}$and the mass difference of proton and neutron. This process has been discussed as a possible source of p-nuclei near A=90 - 100, which have anomalously large isotopic abundances [14].

To investigate this possibility, we need to confirm this process from nuclear physics point of view, as the most nuclear reactions involved are unknown yet. It involves not only proton and alpha induced reactions, but also neutron-induced reactions in the proton-rich unstable nuclear regions. Specifically, the breakout process from the pp-chain region has a quite large influence to the production of nuclei around A 90-100, as can be seen in the sensitivity test shown in Fig. 3. 
The pathway going through ${ }^{7} \operatorname{Be}(\alpha, \gamma){ }^{11} \mathrm{C}$ $(\alpha, p)^{14} \mathrm{~N}$ together with the triple alpha process seem to play a key role. Since this pathway was not known well, we studied the two reactions in the pathway in our project.

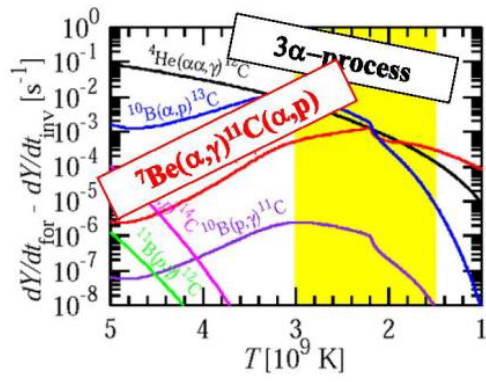

(a)

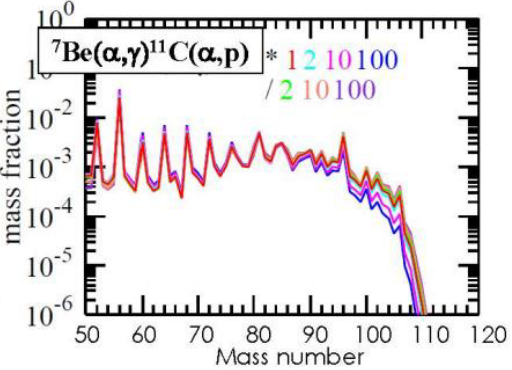

(b) A detail will be discussed in subsec. 2.3.

In addition for the

Figure 3 (a) The breakout reactions from the pp-chain region, important in the early stage of the vp-process [11]. (b) A sensitivity test of ${ }^{7} \mathrm{Be}(\alpha, \gamma){ }^{11} \mathrm{C}(\alpha, p)$ for heavy element synthesis.

$v p$-process, one needs to study neutron-induced stellar reactions on proton-rich nuclei, which is a distinct difference from the ordinary rp-process. But, an important point here is that the vpprocess has a possibility to contribute for the chemical evolution, which is different from the $\mathrm{x}$ ray bursts which do not contribute to it. An interesting consequence of the neutron-induced reactions is that the waiting points of the rp-process may be discarded by the $(n, p)$ and $(n, \alpha)$ reactions, and the nucleosynthesis may flow up to heavier mass regions [11].

The vp-process thus involves nuclear structures which are not investigated yet. The proton capture reactions may be sensitive to low-lying proton resonances of a single particle configuration. Pigmy resonances might also contribute to the $(\mathrm{p}, \gamma)$ reaction if they exist in this energy region, and alpha cluster resonances to the alpha-induced reactions. This possibility is supported by the cluster threshold rule [15], which says that alpha cluster states will appear with a large possibility near the alpha-thresholds. Of course, there are very few experimental studies on alpha cluster resonances in proton-rich unstable nuclear region yet. The neutron thresholds in proton-rich nuclei locate at much higher energy region, where almost no nuclear structure information are available in nuclear physics yet. The neutron-induced reactions on proton-rich unstable nuclei are very difficult to study, as discussed above. If the reactions involve proton-rich nuclei located close to the line of stability, there is a possibility to study it by a time-reverse reactions. If the proton-rich nuclei have a long half-lives, one may make a target for the study. These are, however, very limited, and mostly the proton-rich nuclei relevant are short-lived nuclei located away from the line of stability, which cannot be reached by the time reverse reactions. Currently, people are using statistical models to estimate almost all cross sections of the alpha- and neutron- induced reactions, although statistical models are known to be not precise enough in the light mass regions, and further the statistical models are not investigated so much in unstable nuclear regions. Thus, the experimental studies of the stellar reactions by alpha and neutrons should be very important and a challenge for the coming years in nuclear astrophysics. I will discuss these subjects along with our recent experimental efforts, specifically emphasizing the astrophysical reactions starting from ${ }^{7} \mathrm{Be}$ and ${ }^{7} \mathrm{Li}$ in the following sections.

\section{Fate of ${ }^{7} \mathrm{Be}$}

\section{$2.1{ }^{7} \mathrm{Be}(\mathrm{n}, \alpha)^{4} \mathrm{He}$ reaction}

There is only one direct measurement of the cross sections of ${ }^{7} \mathrm{Be}(\mathrm{n}, \alpha){ }^{4} \mathrm{He}$ reported at thermal energy [16]. As discussed in subsec. 1.1, this rate could be very influential if the rate is higher than the 
current rate adopted [17]. There is one relevant experiment made by the time reverse reaction, ${ }^{4} \mathrm{He}(\alpha, n){ }^{7} \mathrm{Be}$ [18], although there is no data at the temperature region of interest, but show cross sections above the threshold, suggesting a possible extrapolation to the lower energies. In addition, there are many experiments on the mirror reaction, ${ }^{7} \operatorname{Li}(\mathrm{p}, \alpha)^{4} \mathrm{He}$, with which one can make a reasonable estimate of the cross section for ${ }^{7} \mathrm{Be}(\mathrm{n}, \alpha)^{4} \mathrm{He}$ by correcting for the penetrabilities for the Coulomb barriers as well as for the centrifugal barriers as well as the exact excitation energies. The estimated cross sections [19] shown in Fig. 4 are roughly a factor of 10 smaller than the Wagoner rate currently adopted [17]. The BBN simulation shows that the present rate leads to an increase of $1.2 \%$ of the ${ }^{7} \mathrm{Li}$ abundance, compared with the result using the Wagoner rate, and thus, the new rate makes the ${ }^{7} \mathrm{Li}$ problem worse. The present estimate should be confirmed by experiment.

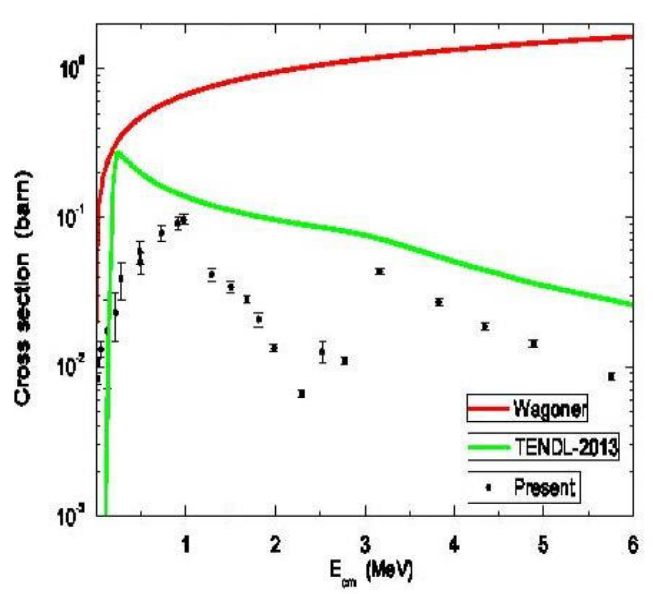

Figure 4 The cross sections of the ${ }^{7} \mathrm{Be}(\mathrm{n}, \alpha)^{4} \mathrm{He}$ reaction estimated from other reaction data together with the cross section at the thermal energy [19].

\section{$2.2{ }^{7} \mathrm{Be}(\alpha, \gamma)^{11} \mathrm{C}$ reaction}

The nucleosynthetic flows through the proton-rich nuclear side in the pp-chain region are considered to affect significantly the production of heavy nuclei in the vp-process [11], as discussed in subsec. 1.3. At extremely high temperatures, alpha induced reactions are expected to play a key role in the light mass regions.

Among the possible breakout pathways from the pp-chain region, the least investigated but very crucial pathway is ${ }^{7} \mathrm{Be}(\alpha, \gamma){ }^{11} \mathrm{C}(\alpha, p){ }^{14} \mathrm{~N}$. The first step reaction ${ }^{7} \mathrm{Be}(\alpha, \gamma){ }^{11} \mathrm{C}$, which is considered to have the decisive role in the pathway, was studied previously by the direct method using a ${ }^{7} \mathrm{Be}$ target [20], but it was limited up to the second resonance above the $\alpha$-threshold. We need to know the contributions from the high-lying resonances in ${ }^{11} \mathrm{C}$, which might have significant contributions at high temperatures.

In order to study the highlying resonances, an experiment was performed [21] for ${ }^{7} \mathrm{Be}+\alpha$ resonant scattering with a thick-target method using a high-intensity, nearly pure ${ }^{7} \mathrm{Be}$ beam from the CRIB facility $[22,23]$ of the University of Tokyo, set in the RIKEN RIBF facility. The ${ }^{7} \mathrm{Be}$ beam was about $2 \times 10^{5}$ pps and $17.9 \mathrm{MeV}$. Both elastic and inelastic events were measured separately by using a $\Delta \mathrm{E}-\mathrm{E} \mathrm{Si}$ detector telescope together with

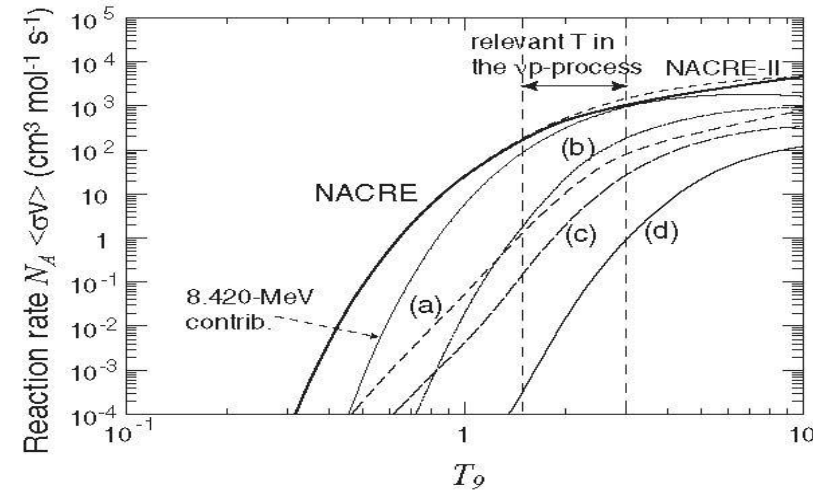

Figure 5 The reaction rates of ${ }^{7} \operatorname{Be}(\alpha, \gamma){ }^{11} \mathrm{C}$, deduced based on the present experiment [21]. The curves (a) and (b) represent the contribution of the newly found, third resonance, assuming $9 / 2^{+}$ and $3 / 2^{+}$, respectively. 
$10 \mathrm{NaI}(\mathrm{Tl})$ detectors for particle identifications.

The elastic and inelastic scattering of $\alpha+{ }^{7} \mathrm{Be}$ were measured together with the ${ }^{7} \mathrm{Be}(\alpha, \mathrm{p})$ reaction. A new resonance at $8.90 \mathrm{MeV}$, which is the third resonance above the alpha threshold, was identified for the first time, with possible spin-parity assignment of $\left(9 / 2^{+}\right)$or $3 / 2^{+}$, where the R-matrix analysis did not give a unique assignment [21]. New rotational bands were identified that have a large alpha configuration.

The reaction rates estimated through this resonance are shown in Fig. 5. Here, the gamma decay width of this state was simply assumed to be a typical value, 0.1 of the Weisskopf unit. This work suggests about $10 \%$ increase of the total reaction rate of ${ }^{7} \mathrm{Be}(\alpha, \gamma){ }^{11} \mathrm{C}$ due to the new third resonance above the alpha threshold in ${ }^{11} \mathrm{C}$. Since this reaction rate is considered to have the decisive role for breakout from the pp-chain region, this gamma width needs to be investigated experimentally.

\section{$2.3{ }^{11} \mathrm{C}(\alpha, \mathrm{p}){ }^{14} \mathrm{~N}$ reaction}

The second reaction ${ }^{11} \mathrm{C}(\alpha, \mathrm{p}){ }^{14} \mathrm{~N}$ on the breakout process was studied recently [24] by the direct method for the first time using a high-intensity ${ }^{11} \mathrm{C}$ beam at very low energies. This reaction was previously studied only by the time-reverse reaction ${ }^{14} \mathrm{~N}(\mathrm{p}, \alpha){ }^{11} \mathrm{C}$ with an activation method.

Apparently, the time reverse reaction study is not sensitive to the reactions leading to the excited states in ${ }^{14} \mathrm{~N}$. It should be also worthwhile to confirm by the direct method the cross sections to the ground state derived previously by the activation method.

The ${ }^{11} \mathrm{C}(\alpha, \mathrm{p}){ }^{14} \mathrm{~N}$ experiments[24] were performed at the CRIB facility [22,23]. The ${ }^{11} \mathrm{C}$ beam at low energies were produced at the CRIB facility; $3 \times 10^{5}$ pps at $10.1 \mathrm{MeV}$, and $1 \times 10^{5} \mathrm{pps}$ at 16.9 $\mathrm{MeV}$ with a purity better than $97 \%$. A new method was developed here for efficient measurement of the excitation functions of the ${ }^{11} \mathrm{C}(\alpha, p){ }^{11} \mathrm{~N}$ reaction cross sections for the lowlying excited states as well as for the ground state in ${ }^{14} \mathrm{~N}$. We adopted a thick-target method with an extended gas target together with two position sensitive beam monitors, and position sensitive Si telescopes for the measurement of protons. By combining the TOF information, one can identify the reaction points in the target, and thus the reaction energies of the $(\alpha, p)$ reaction. Precise excitation functions were obtained for the ${ }^{11} \mathrm{C}(\alpha, \mathrm{p}){ }^{11} \mathrm{~N}$ reactions for the ground states and the first and second excited states [24]. The cross sections for the ground state transitions obtained agree well with the cross sections previously measured by the time-reverse reaction with the activation method [25]. The cross sections for the first and second excited states were also obtained for the first time, and our data consistently disagree with the statistical model predictions roughly by a factor of 2-5.

The R-matrix analysis identified several new resonances, and new resonance parameters for the known states in ${ }^{14} \mathrm{~N}$. This is the first extensive, direct measurement of the stellar $(\alpha, p)$ reaction cross sections with the proton-rich unstable nuclei, identifying each resonance and the resonance parameters. It is interesting to note that alpha cluster states enhance the reaction rates; some large peaks of the reaction cross sections are due to large alpha widths, as expected.

This experiment has successfully determined for the first time the ${ }^{11} \mathrm{C}(\alpha, \mathrm{p}){ }^{11} \mathrm{~N}$ reaction rates at the temperature region important for the $v p$-process, including the transitions to the excited states in ${ }^{14} \mathrm{~N}$. The present result supports the vp-process simulation that nucleosynthesis-flow runs up to the mass region of $A=90 \sim 100$. This study also has confirmed very precisely the cross sections for the ${ }^{11} \mathrm{C}\left(\alpha, \mathrm{p}_{0}\right){ }^{11} \mathrm{~N}$ (g.s.) reaction by the time-reverse reaction with the activation method. 


\section{Fate of ${ }^{7} \mathrm{Li}$}

\section{1 ${ }^{7} \mathrm{Li}(n, \gamma)^{8} \mathrm{Li}$ reaction}

Possible heavy elements synthesis in the inhomogeneous big bang model was discussed seriously late 1980s and 1990s both in theory [12] and experiment [26-33]. One of the important pathway suggested was ${ }^{7} \mathrm{Li}(\mathrm{n}, \gamma){ }^{8} \mathrm{Li}(\alpha, \mathrm{n}){ }^{11} \mathrm{~B}$ for the $\mathrm{CNO}$ element synthesis. Later, this pathway also has been identified to be important in the type II supernovae in the early stage of the r-process. The ${ }^{7} \mathrm{Li}(\mathrm{n}, \gamma)^{8} \mathrm{Li}$ reaction is the first step of this breakout process.

A new experiment on this reaction [26] reported significantly smaller cross sections than reported earlier [27], roughly by a factor of two, suggesting serious change to the scenario, where a new activation method was applied. We studied the reaction by directly detecting the gamma rays from the reaction [28], and the cross sections obtained is quite consistent with the old measurement [27] but not with the "new" data [26]. There are several, extensive follow-up experiments reported, all supporting the old data [27]. In addition, the group who reported the "new data" made another experiment and found an agreement with the old data [27] and also with other follow-up experiments [29]. Some theoretical works later took an average of the data sets of this reaction including the erroneous data [26], which should be carefully avoided.

Thus, this reaction is one of the few reactions whose cross sections are now well known by experiment for a wide temperature range.

\section{$3.2{ }^{8} \mathrm{Li}(\alpha, \mathrm{n})^{11} \mathrm{~B}$ reaction}

This reaction is the second reaction on the breakout process of ${ }^{7} \operatorname{Li}(n, \gamma)^{8} \operatorname{Li}(\alpha, n){ }^{11} \mathrm{~B}$. The first experiment was made by the time reverse reaction ${ }^{11} \mathrm{~B}(\mathrm{n}, \alpha)^{8} \mathrm{Li}$ with the activation method [30]. This experiment is just sensitive to the ground state transition in ${ }^{8} \mathrm{Li}(\alpha, n){ }^{11} \mathrm{~B}(\mathrm{~g} . \mathrm{s}$.), although there are several excited states below the particle thresholds in ${ }^{11} \mathrm{~B}$. The decay property was investigated by the ${ }^{9} \mathrm{Be}(\alpha, p){ }^{12} \mathrm{~B}(\mathrm{n}){ }^{11} \mathrm{~B}^{*}$ reaction [31]. The intensities to the excited states amount to about the same intensity as to the ground state. However, the resonances excited by this $(\alpha, p)$ reaction was not so clear if they are the same one seen in the ${ }^{11} B(n, \alpha)$ reaction, as the width seems significantly different.

Soon after, a radioactive beam of ${ }^{8} \mathrm{Li}$ became available, which enabled a direct measurement of the reaction cross sections using a ${ }^{8} \mathrm{Li}$ beam on ${ }^{4} \mathrm{He}$ target. The first experiment was made at RIKEN, although the ${ }^{8} \mathrm{Li}$ beam was not so good at the time [32]. A few experiments followed using better ${ }^{8} \mathrm{Li}$ beams and better experimental setups. Figure 6 shows the most recent work together with the results of the preceding works [33]. It already covers partly the important temperature range. There seem, however, some resonances locating in the temperature region of interest, but the data still show a large scatter, as can be seen in Fig. 6. The lower temperature region also needs to be investigated as they are effective at around $1 \mathrm{GK}$ and below. It should be of great interest to pursue this subject down to $0.5 \mathrm{GK}$ with a good counting statistics.

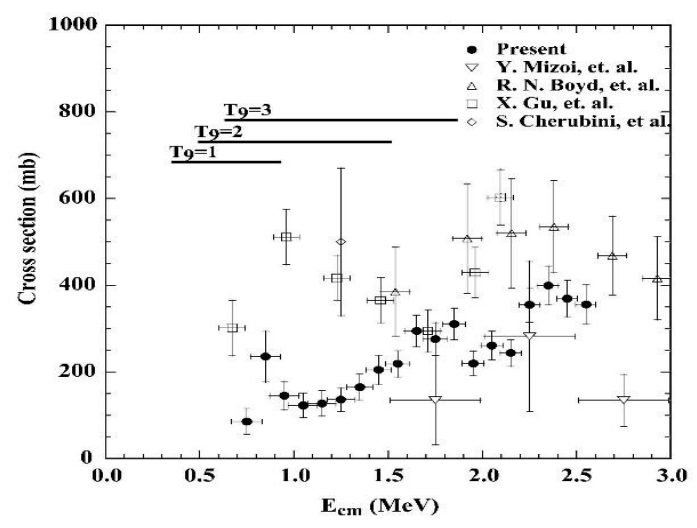

Figure 6 The reaction rate of ${ }^{8} \operatorname{Li}(\alpha, n){ }^{11} \mathrm{~B}$, deduced based on the present experiment [33], together with other data indicated. 


\section{Summary}

The important nuclear reactions at high temperature conditions in the pp-chain region, and on the possible breakout process were discussed. Specifically, the reactions relevant to ${ }^{7} \mathrm{Li}$ and ${ }^{7} \mathrm{Be}$ were discussed including our experimental efforts. These reactions are the neutron- and alpha- induced reactions, and most of them are poorly understood, awaiting experimental efforts in order to study the primordial nucleosynthesis and type II supernovae.

\section{References}

[1] Proc. Origin of Matter and Evolution of Galaxies 2011, eds. S. Kubono, et al., AIP Conf. Proc. 1484 (2012). Proc. Origin of Matter and Evolution of Galaxies 2011, eds. S. Jeong, et al., AIP Conf. Proc. 1594 (2013). Proc. XII International Symposium on Nuclei in the Cosmos, Cairns, 2012, eds. J. Lattanzio, et al. Proceedings of Science (NIC XII), S. Kubono, Nucl. Phys. A 693 , $221(2001)$

[2] J. Dunkley, et al., Astrophys. J., Suppl. Ser. 180, 306 (2009).

[3] Plank Collaboration, 2015, arXiv:1502.01582 (2015)

[4] F. Spite, M. Spite, Astron. Astrophys. 115 (1982) 357

[5] B. D. Fields, Annu. Rev. Nucl. Part. Sci. 61, 47 (2011)

[6] C. Fröhlich, et al., Phys. Rev. Lett. 96, 142502 (2006)

[7] J. Pruet, et al., Astrophys. J. 644, 1028 (2006)

[8] S. Wanajo, Astrophys. J. 647, 1323 (2006)

[9] R. K. Wallace and S. E. Woosley, Astrophys. J. Suppl. 45, 389 (1981)

[10] S. Q. Hou, et al., Chin. Phys. Lett. 27, 082601 (2010)

[11] S. Wanajo, H.-T. Janka, and S. Kubono, Astrophys. J. 729, 46 (2011)

[12] J.H. Applegate, C. J. Hogan, and R.J. Scherrer, Phys. Rev. D 35 (1988) 1151, T. Kajino, G.J. Mathews, G. M. Fuller, Astrophys. J. 364 (1990) 7

[13] T. Sasaqui, et al., Astrophys. J. 645 (2006) 1345

[14] M. Arnould, and S. Goriely, Phys. Rep. 384 , 1 (2003)

[15] S. Kubono, et al., 10th International Conference on Clustering Aspects of Nuclear Structure and Dynamics, IOP Conf. 436, 012071 (2013)

[16] E. P. Bassi et al., Nuovo Cimento 28, 1049 (1963)

[17] R. V. Wagoner, Astrophys. J., Suppl. Ser. 18, 247 (1969).

[18] C. H. King, et al., Phys. Rev. C 16 (1977) 1712

[19] S.Q. Hou, J.J. He, S. Kubono, and Y.S. Chen, Pys. Rev. C 91 (2015) 055802

[20] G. Hardie, et al., Phys. Rev. C 29 (1984) 1199

[21] H. Yamaguchi, et al., Phys. Rev. C 87, 034303 (2013).

[22] S. Kubono, et al., Eur. Phys. J. A 13, 217 (2002)

[23] Y. Yanagisawa, S. Kubono, et al., Nucl. Instr. Meth. A 539, 74 (2005)

[24] S. Hayakawa, Ph. D. thesis of the University of Tokyo, 2012.

[25] P.D. Ingalls, et al., Phys. Rev. C 13 (1976) 524

[26] M. Wiescher, et al., Astrophys. J. 344 (1989) 464

[27] W. Imhof, et al., Phys. Rev. 14 (1959)1037

[28] Y. Nagai, et al., Astrophys. J. 381 (1991) 444

[29] M. Heil, et al., Astrophys. J. 507 (1998) 997

[30] T. Paradellis, et al., Z. Phys. A 337 (1990) 211

[31] S. Kubono, et al., Z. Phys. A 338 (1991) 459, S. Kubono, et al., Z. Phys. A 341 (1991) 121, R. Boyd, S. Kubono, et al., Nucl. Phys. A 542 (1992) 97

[32] R.N. Boyd, et al., Phys. Rev. Lett. 68 (1992) 1283

[33] H. Ishiyama, et al., Phys. Lett. B 640 (2006) 82 